\title{
Is attention needed for word identification? Evidence from the Stroop paradigm
}

\author{
JOEL LACHTER \\ NASA Ames Research Center, Moffett Field, California \\ and San Jose State University, San Jose, California \\ ERIC RUthrufF \\ University of New Mexico, Albuquerque, New Mexico \\ Mei-Ching Lien \\ Oregon State University, Corvallis, Oregon \\ AND \\ Robert S. MCCANN \\ NASA Ames Research Center, Moffett Field, California
}

\begin{abstract}
One of the most robust findings in attention research is that the time to name a color is lengthened markedly in the presence of an irrelevant word that spells a different color name: the Stroop effect. The Stroop effect is found even when the word is physically separated from the color, apparently indicating that words can be read outside the focus of spatial attention. The present study critically evaluated this claim. We employed several stringent measures within a Stroop paradigm to prevent participants from attending to the irrelevant words (e.g., limiting exposure duration to prevent attention capture). Nonetheless, residual Stroop effects were obtained for both color words and semantic associates (e.g., sky to blue). These data suggest that lexical processing can sometimes occur outside the focus of spatial attention.
\end{abstract}

Attention is the mechanism that allows people to select, from among the large set of stimuli to which they could possibly respond, the small number to which they actually do respond. Although the need for such selection is clear, there is also a countervailing need to identify other items in the environment because they may present dangers or opportunities. How much processing of such items takes place prior to attentional selection? Although many researchers have attempted to answer this fundamental question, different methodologies have led to different conclusions. In particular, the results of many studies using Stroop paradigms have suggested that words are identified without attention, whereas the results of studies using lexical-decision paradigms have led to the opposite conclusion (see Brown, 1996).

In the traditional Stroop paradigm, participants are asked to name, as rapidly as possible, the color of the ink in which a word is written (Stroop, 1935; see MacLeod, 1991, for a review). If the word spells the name of the ink color (e.g., GREEN printed in green ink), responses are quick and accurate. If, however, the word spells a different color (e.g., RED printed in green ink), responses are relatively slow and error prone. This influence of word identity on response time (RT) has been found even in designs in which the word is never congruent with the color (see, e.g., Stroop, 1935); this finding demonstrates that the words are identified even when doing so is always detrimental to task performance. Importantly for the present purposes, Stroop effects occur even when the color word is in a fixed location several degrees away from the target stimulus whose color is to be named (see MacLeod, 1991). Findings of this type have been used to argue that words outside the focus of spatial attention can be identified (e.g., Brown, Gore, \& Carr, 2002; Neely \& Kahan, 2001).

The conclusion that words are identified outside the focus of spatial attention has recently been challenged. Lachter, Forster, and Ruthruff (2004) noted that the experimental conditions used in most Stroop paradigms would allow attention to "slip" to the irrelevant word (the attentional slip hypothesis). They argued that irrelevant objects (especially those that appear abruptly) could attract attention to themselves within $100 \mathrm{msec}$ of their appearance. Color words in Stroop paradigms usually are present for more than $100 \mathrm{msec}$ and are not masked (allowing processing of the iconic image). Thus, there is plenty of time for these words to attract attention to themselves before they are extinguished. 
Using repetition priming in a lexical-decision paradigm (i.e., the participants' task was to determine whether letter strings formed words or not), Lachter et al. (2004) looked for evidence that unattended words could be identified without attentional slippage. Their paradigm was analogous to the Stroop paradigm in that they measured the effects of the congruence of irrelevant words on RT to the target. The irrelevant words could be the same as the target or unrelated to the target, allowing the authors to measure repetition priming. Several steps were taken to prevent attention from slipping to these irrelevant distractor words. First, both exogenous and endogenous cues were used, in order to ensure that attention was directed to the target-word location but not to the distractor-word location. Second, distractor words were masked after only $55 \mathrm{msec}$, so attention could not shift to them in time to facilitate processing. Under these conditions, masked distractor words produced no detectable repetition-priming effect on lexical decisions. A control condition, however, showed that the same brief, masked words, when they were spatially attended, could strongly affect lexical decisions. This conclusion is also supported by a spatial-cuing study conducted by McCann, Folk, and Johnston (1992), the results of which suggested that unattended words could not be identified until after attention had shifted to their location.

Given Lachter et al.'s (2004) findings with lexicaldecision paradigms, it is reasonable to suspect that Stroop effects occur only because the color words are often attended (slippage of attention). However, there are several reasons why the Stroop paradigm might be better able to detect the effects of lexical processing without attention (leakage through the attentional filter) than the lexicaldecision paradigm. First, the large size and robust nature of the Stroop effect may allow one to see residual effects even under manipulations (such as removal of attention) expected to reduce effect sizes. Second, the same color words are presented many times throughout the experiment in the Stroop paradigm, whereas each word is usually presented only once in the lexical-decision paradigm. Repeatedly presented words might be more activated and thus more easily recognized. Finally, in the Stroop paradigm, it might be relatively easy to process the irrelevant color word in parallel with the target colored object because the object and the word do not compete for the same resources. In contrast, the unattended prime word in Lachter et al.'s lexical-decision paradigm competed directly with the target word for word-processing resources. For instance, the perceptual features of the attended target word might interfere with the perceptual features of the unattended prime word (see Brown et al., 2002, and Brown, Roos-Gilbert, \& Carr, 1995, for an account of Stroop dilution based on this assumption).

To resolve these issues, we examined whether the Stroop effect would persist even when extreme precautions were taken to prevent attentional slippage (i.e., to ensure that the words were spatially unattended). On the basis of our previous research with lexical-decision tasks, we expected that preventing attentional allocation to the words would eliminate the Stroop effect.

\section{EXPERIMENT 1}

Experiment 1 was designed to determine whether the Stroop effect would be eliminated when the color words were not attended. Participants named the color of a filled rectangle (bar) in the presence of an adjacent color word. We took several steps to ensure that participants attended the bar and not the word: (1) The bar was always presented at the same central location (with words presented above or below it); ${ }^{1}$ (2) premasks were used, so that attention could be allocated precisely (endogenously) to the bar location; (3) a precue was used to capture attention exogenously to the bar location; (4) the word was presented for only $50 \mathrm{msec}$ and then masked before it could capture attention (attention shifts are thought to require $>50 \mathrm{msec}$ ); and (5) an irrelevant color band was placed around the target color bar, to encourage a tight attentional focus. These precautions closely followed those used by Lachter et al. (2004) in their lexical-decision-priming paradigm. Although one or two of these precautions have been used in other Stroop studies (e.g., Brown et al., 2002; Cho, Lien, \& Proctor, 2006), those studies did not guard against all possible causes of attentional slippage (see Lachter et al., 2004). Importantly, despite the brief exposures of the words, pilot studies showed that they produce substantial Stroop effects when spatially attended ( $\sim 30 \mathrm{msec}$; see also Lachter et al., 2004). ${ }^{2}$

\section{Method}

Participants. A total of 24 students from colleges and universities surrounding NASA's Ames Research Center participated in exchange for extra course credit. All participants had normal or corrected-to-normal vision.

Apparatus, Stimuli, and Design. The stimuli were presented on 20 -in. Sony Trinitron monitors. There were three rows of stimuli, centered $1.6 \mathrm{~cm}$ apart. The top and bottom rows contained characters displayed in bold Helvetica typeface; uppercase letters had a height of $1.0 \mathrm{~cm}$. The central row contained a rectangle measuring $4.8 \mathrm{~cm}$ wide $\times 1.0 \mathrm{~cm}$ high. At a typical viewing distance of $60 \mathrm{~cm}$, the stimulus subtended a visual angle of $4.6^{\circ}$ wide and $1.0^{\circ}$ high. The irrelevant color word, presented in white in the top or bottom row, was the lowercase word blue, green, red, or yellow. Each target color was paired equally often with each color word, so that the target and color word were congruent on $25 \%$ of the trials and incongruent on the remaining $75 \%$.

Procedure. Each trial began with a fixation cross in the center of the screen for $500 \mathrm{msec}$ followed by a $300-\mathrm{msec}$ blank interval. Then the string XXXXXX appeared in the top and bottom rows and a white border appeared around the center box, as is shown in Figure 1. After $500 \mathrm{msec}$, the center box filled briefly with each of the four target colors (for $25 \mathrm{msec}$ each), in a random sequence, to capture attention to the center location. Next, the center bar was again unfilled and the irrelevant color word was presented for $50 \mathrm{msec}$ either above or below the central location. Finally, the target appeared in the center box for $100 \mathrm{msec}$. The target consisted of a central color bar, which participants were asked to name, surrounded by a $0.25-\mathrm{cm}$-thick border. The irrelevant border color never matched the target color or the irrelevant color word. Simultaneously with the target, the string "\#\$\%?@\&" appeared for $600 \mathrm{msec}$ in both the top and bottom rows to mask the word.

Participants were to name the color of the target bar by saying "blue," "green," "red," or "yellow" into a microphone. After participants responded (or after a 5-sec time-out period elapsed), response accuracy was determined by the computer, and visual 


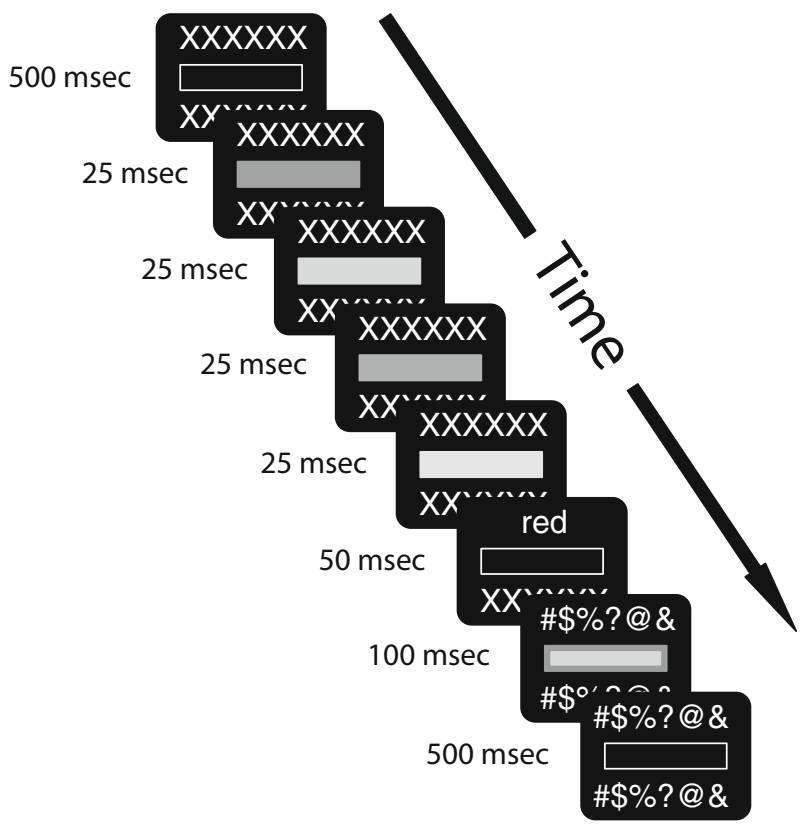

Figure 1. An example of the stimuli used in these experiments showing the time course. The center box, here shown in grayscale, was colored in the actual experiments.

feedback was presented for $300 \mathrm{msec}$ ("Correct" for correct responses, "Incorrect" for incorrect responses, "Too late" for timeouts, or "Unknown" for unrecognized responses). The next trial began $700 \mathrm{msec}$ after feedback offset. The participants performed one practice block of 40 trials and five regular blocks of 90 trials each. The experiment lasted approximately $25 \mathrm{~min}$. The participants were told that both speed and accuracy were very important. After each block, participants received a summary of their average RT and accuracy for that block.

\section{Results and Discussion}

Trials were eliminated if the RT was outside the range of $200-1,500 \mathrm{msec}(0.4 \%$ of trials). For RT analyses, trials were also omitted if the current or previous response was an error. Data were analyzed as a function of the congruency between the word and the target color (congruent vs. incongruent).

Table 1 shows the mean RTs and percentages of error (PEs) for the congruent and incongruent conditions. Contrary to our expectations, there was a significant 14-msec Stroop (congruency) effect on RT $[F(1,23)=12.94, p<$ $.01]$. The trend for PE was in the same direction but was not statistically significant $[F(1,23)=1.95, p=.1755]$.

Despite the extreme measures taken to prevent participants from attending the words, a Stroop effect remained. This finding contradicts the attentional slip hypothesis as the sole explanation of Stroop interference when the color and word are spatially separated. The results contrast sharply with those of Lachter et al. (2004), who took essentially the same extreme measures to control the locus of attention in a lexical-decision paradigm and found no repetition priming from unattended words.

\section{EXPERIMENTS 2A AND 2B}

In Experiment 1, we demonstrated that some aspect of an unattended color word could be identified. However, it was not necessarily the case that participants identified the color word as a whole. Because the color words were used many times throughout the experiment, it was possible that the effects seen in Experiment 1 resulted from the participants' identifying only a few letters, which then might have directly activated the phonology necessary to pronounce a verbal response.

We conducted Experiments 2A and 2B in order to determine whether effects similar to those found in Experiment 1 could be generated even with noncolor words (Experiment 2A) and nonwords (Experiment 2B) that shared only a few letters with the associated color. Such a finding would be consistent with a partial analysis of the word and would thus call into question the claim that words are pro-

Table 1

Mean Response Times (RTs, in Milliseconds) and Percentages of Error (PEs)

for Incongruent and Congruent Trials, Along With the Stroop Effect in Experiments 1-3

\begin{tabular}{|c|c|c|c|c|c|c|c|c|c|c|c|c|}
\hline \multirow[b]{4}{*}{ Distractor Type } & \multicolumn{8}{|c|}{ Congruency } & & & & \\
\hline & \multicolumn{4}{|c|}{ Incongruent } & \multicolumn{4}{|c|}{ Congruent } & \multicolumn{4}{|c|}{ Stroop Effect } \\
\hline & \multicolumn{2}{|c|}{ RT } & \multicolumn{2}{|c|}{$\mathrm{PE}$} & \multicolumn{2}{|c|}{ RT } & \multicolumn{2}{|c|}{$\mathrm{PE}$} & \multicolumn{2}{|c|}{ RT } & \multicolumn{2}{|c|}{$\mathrm{PE}$} \\
\hline & $M$ & $S E$ & $M$ & $S E$ & $M$ & $S E$ & $M$ & $S E$ & $M$ & $S E$ & $M$ & $S E$ \\
\hline \multicolumn{13}{|c|}{ Experiment 1} \\
\hline Color word & 567 & 16 & 3.2 & 0.7 & 553 & 16 & 2.5 & 0.7 & 13.7 & 3.8 & 0.7 & 0.5 \\
\hline \multicolumn{13}{|c|}{ Experiment $2 \mathrm{~A}$} \\
\hline Color word & 586 & 11 & 3.4 & 0.3 & 577 & 12 & 2.8 & 0.5 & 9.9 & 3.3 & 0.6 & 0.4 \\
\hline Orthographically related word & 587 & 12 & 2.8 & 0.4 & 579 & 13 & 3.3 & 0.4 & 8.6 & 3.1 & -0.5 & 0.3 \\
\hline \multicolumn{13}{|c|}{ Experiment 2B } \\
\hline Color word & 579 & 12 & 4.0 & 0.5 & 563 & 12 & 3.7 & 0.5 & 16.1 & 4.1 & 0.3 & 0.5 \\
\hline Orthographically related nonword & 576 & 12 & 4.2 & 0.6 & 566 & 12 & 3.8 & 0.6 & 10.0 & 3.2 & 0.4 & 0.5 \\
\hline \multicolumn{13}{|c|}{ Experiment 3} \\
\hline Color word & 566 & 9 & 4.1 & 0.3 & 562 & 9 & 4.0 & 0.3 & 4.2 & 1.2 & 0.1 & 0.3 \\
\hline
\end{tabular}

Note-Color words were red, green, blue, and yellow. Orthographically related words were rent, greasy, bloom, and yes. Orthographically related nonwords were rell, bloot, greamy, and yem. Semantically related words were sky, navy, cherry, crimson, lime, pea, lemon, and mustard. Stroop Effect = Incongruent - Congruent. 
cessed as a whole. Because these noncolor words and nonwords were chosen to share initial letters with the color words, we refer to them as orthographically related.

\section{Method}

Participants. There were 38 new participants in each experiment. Participants in Experiment 2A were recruited from colleges and universities surrounding NASA's Ames Research Center and from Oregon State University. Participants in Experiment 2B were recruited from colleges and universities surrounding NASA's Ames Research Center and from the University of New Mexico. All participated in exchange for extra course credit.

Apparatus, Stimuli, and Procedure. The tasks, stimuli, and equipment were identical to those in Experiment 1, except as follows. On half the trials in Experiment 2A, the color word used in Experiment 1 was replaced by an orthographically related word: rent, greasy, bloom, or yes. On half of the trials in Experiment 2B, the color word was replaced by an orthographically related nonword: rell, bloot, greamy, or yem. In both cases, these stimuli were classified as congruent if their initial letters were phonetically consistent with the name of the target color (e.g., rent and rell are congruent with the color red)

\section{Results and Discussion}

As in Experiment 1, we eliminated trials with an RT less than $200 \mathrm{msec}$ or greater than $1,500 \mathrm{msec}(0.2 \%$ of trials). Data were analyzed as a function of congruency (congruent vs. incongruent) and stimulus type (color word vs. orthographically related).

Table 1 shows the mean RTs and PEs across conditions. In Experiment 2A, there was a significant Stroop effect on RT $[F(1,37)=12.89, p<.001]$. The Stroop effect was $9.9 \mathrm{msec}$ when the word was a color name and $8.6 \mathrm{msec}$ when it was an orthographically related word. Separate analyses on the two stimulus types revealed that the Stroop effect was significant both for color words $[F(1,37)=8.93, p<.01]$ and for orthographically related words $[F(1,37)=7.66, p<.01]$. Neither the main effect of stimulus type nor its interaction with congruency was significant $\left[F_{\mathrm{s}}(1,37)<1\right]$. For $\mathrm{PE}$, no effects were significant.

Similarly, in Experiment 2B, there was a significant Stroop effect on RT $[F(1,37)=23.67, p<.001]$. The Stroop effect was $16.1 \mathrm{msec}$ when the word was a color word and $10.0 \mathrm{msec}$ when it was an orthographically related nonword. Separate analyses on the two stimulus types revealed that the Stroop effect was significant for both color words $[F(1,37)=15.32, p<.001]$ and orthographically related nonwords $[F(1,37)=9.45, p<.01]$. The main effect of the stimulus type was not significant $[F(1,37)<1]$, nor was its interaction with congruency $[F(1,37)=1.43]$. For PE, no effects were significant.

These results from the color-name condition replicated the findings of Experiment 1. Clearly, participants in this paradigm identified some aspect of the unattended stimuli. However, the difference between actual color names and those that shared only a few letters was nonsignificant. Thus, much of the effect found in Experiment 1 could be attributed to letter-level identification rather than to whole-word identification.

\section{EXPERIMENT 3}

Although both Experiments 2A and 2B suggested that much of the Stroop effect with unattended words could be attributed to identification of only the first few letters, they fell short of demonstrating that the words were never identified as a whole. In Experiment 3, we looked for direct evidence that words could be identified as wholes without attention. To accomplish this goal, we used words that were semantically related to the reported color names but had no systematic orthographic relationship to those color names (sky, navy, cherry, crimson, lime, pea, lemon, and mustard). Because the individual letters bore no relationship to the colors, any Stroop effect found here would have to be mediated by whole-word processing.

\section{Method}

Participants. There were 96 new participants from Oregon State University and the University of New Mexico.

Apparatus, Stimuli, and Procedure. The tasks, stimuli, and equipment were the same as those in Experiments 2A and 2B, except that the words were replaced with sky and navy (congruent with blue), cherry and crimson (congruent with red), lime and pea (congruent with green), and lemon and mustard (congruent with yellow).

\section{Results and Discussion}

As in the previous experiments, trials with an RT less than $200 \mathrm{msec}$ or greater than $1,500 \mathrm{msec}(0.5 \%$ of trials $)$ were eliminated. Data were analyzed as a function of congruency between the word and the target color (congruent vs. incongruent).

Table 1 shows mean RTs and PEs. For RT, there was a significant 4.2-msec Stroop effect $[F(1,95)=12.10, p<$ $.001]$. For PE, the Stroop effect trended in the same direction but was not statistically significant $(F<1)$. Although the RT effect was quite small, it was obtained despite a large set of factors working against getting any effect at all (lack of spatial attention, brief exposures, and a merely semantic relationship between color and word). Furthermore, the RT effect was remarkably consistent across the different stimuli. Table 2 shows mean normalized RTs for each combination of target color and word (relative to the mean for that target color). RTs for congruent stimuli are shaded. Table 2 also shows the mean Stroop effect generated by each word and each target color. The Stroop effect was positive for every target color (the column totals) and for every word (the row totals), indicating that the results could not be explained simply by any accidental property of the words (e.g., orthographic overlap with one of the color words).

These data indicate that, in a Stroop task, some lexicallevel processing can occur without attention. There is no physical property of the word crimson that could cause it to activate the response red, nor are the individual letters more likely to activate red than, for example, green. The only relationship between the words and the target colors is semantic association, and access to such semantic association requires whole-word processing. 
Table 2

Normalized Response Times (RTs, in Milliseconds) for Each Combination of Target Color and Word

\begin{tabular}{|c|c|c|c|c|c|}
\hline \multirow[b]{2}{*}{ Word } & \multicolumn{4}{|c|}{ Target Color } & \multirow[b]{2}{*}{ Stroop Effect } \\
\hline & Blue & Red & Green & Yellow & \\
\hline Sky & -3.2 & -0.9 & -7.3 & 2.3 & 1.2 \\
\hline Navy & -2.2 & 2.1 & -2.4 & 4.9 & 3.7 \\
\hline Cherry & 4.3 & -0.5 & 6.4 & 1.9 & 4.7 \\
\hline Crimson & 4.1 & -2.9 & 3.6 & 3.1 & 6.5 \\
\hline Lime & 1.3 & -5.2 & -2.9 & -2.7 & 0.7 \\
\hline Pea & 2.3 & 0.3 & -4.8 & -1.0 & 5.3 \\
\hline Lemon & 1.5 & 5.8 & -0.2 & -8.6 & 11.0 \\
\hline Mustard & -8.0 & 1.3 & 7.6 & 0.1 & 0.2 \\
\hline Stroop effect & 3.6 & 2.3 & 5.2 & 5.7 & 4.2 \\
\hline
\end{tabular}

Note-To make it easier to see the interaction of word and color, we have subtracted from each cell the mean RT for the corresponding target color; raw RTs for each cell can therefore be derived by adding the mean RT for that color to the value indicated in the table. Mean RT was $559.0 \mathrm{msec}$ for blue targets, $561.8 \mathrm{msec}$ for red targets, $578.9 \mathrm{msec}$ for green targets, and $562.6 \mathrm{msec}$ for yellow targets. Stroop effects for each row and each column were calculated by subtracting the mean of the congruent cells (shaded) from the mean of the incongruent cells within that row or that column.

\section{GENERAL DISCUSSION}

The present results indicate that some lexical processing of unattended words occurs in the Stroop paradigm. In Experiment 1, we found Stroop effects even after taking extreme measures to prevent the irrelevant words from being attended. These effects were replicated in Experiments $2 \mathrm{~A}$ and $2 \mathrm{~B}$. Although the results of Experiments 2A and 2B suggested that much of this effect could be accounted for by sublexical (letter-level) priming, the results of Experiment 3 demonstrate that there is a residual semantic effect. This residual semantic effect necessarily implies lexical activation of the unattended word. We conclude, therefore, that the Stroop effect is not caused entirely by slippage (attention accidentally allocated to the irrelevant words) as Lachter et al. (2004) suggested, but rather is also a result of leakage (identification without attention).

Given our previous research in the lexical-decision paradigm, we are particularly impressed by the tenaciousness of the Stroop effects in these experiments. However, it is also important to note that these effects are quite small. Had the words been attended, we would have expected effects two to three times larger. Thus, although we were impressed by the robustness of the Stroop effect under the present conditions, those who have argued that spatial attention plays no role whatsoever in word reading should be equally impressed that the effect is not bigger.

These results appear to contradict those of Besner and Stolz (1999), who found that when attention was focused on a particular letter in a word, the word as a whole did not generate a Stroop effect. From this result, Besner and Stolz argued that attention is capable of suppressing the processing of whole words completely. Neely and Kahan (2001) suggested an explanation for this result that fits with the present data. They noted that, in the Besner and Stolz paradigm, participants must use attention to divide the word into figure (the target letter) and ground. They suggested that this division, rather than the lack of spatial attention, prevented the word from being identified.

These findings also appear to contradict Lachter et al. (2004), who found no evidence for lexical processing of unattended words. Reconciling these results is clearly a major research project. Here, we outline four categories of potential explanations: sampling error, continued slippage, stimulus differences, and task differences. First, the difference in results might be due to chance, such as Type I or Type II error, or to differences in the participant pools. This category of explanation is difficult to rule out without extensive replication; however, we have conducted additional, unpublished replications in each of these paradigms, making Type I or Type II error unlikely. ${ }^{3}$

Second, it is possible that a small amount of slippage occurred here despite our precautions. However, it seems unlikely that more slippage occurred here than in Lachter et al.'s (2004) Experiment 5, which used essentially the same precautions. Furthermore, Lachter et al. employed a repetition-priming paradigm because, in pilot work, masked words produced a larger repetition-priming effect than Stroop effect when attended. Thus, if slippage caused the Stroop effects found here, we would have expected it to cause repetition-priming effects under the similar conditions employed by Lachter et al.

Third, there were many physical differences between the stimuli presented here and those used by Lachter et al. (2004). For instance, Lachter et al. presented black stimuli against a white background, whereas in the present experiments we presented stimuli white on black (to improve the visibility of the colors). Although such differences may seem trivial, they have occasionally proven to be important in other paradigms (e.g., Fera, Jolicœur, \& Besner, 1994).

A fourth category of explanation relates to differences between the Stroop and lexical-decision tasks (see Brown, 1996). In the Stroop task, the same colors are presented repeatedly, which presumably allows them to become highly primed and activated. Therefore, these concepts might be reactivated even with very meager stimulus processing. In a lexical-decision task, on the other hand, words are usually presented only once in a session. It may be that, as originally suggested by Treisman (1960), attention attenuates stimulus processing, but not enough to prevent some lexical processing of already highly activated words. Note that, in Experiment 3, although the color associates were not themselves named, they were semantically associated with colors that were highly activated, and thus could have became activated indirectly.

Another difference between the Stroop and lexicaldecision tasks is that, in the Stroop task, participants identify colors while ignoring words, whereas in the lexical-decision task they identify words. Perhaps when the resources required to identify words are fully allocated to processing the target (as in lexical decision), they cannot process unattended words. In the Stroop task, meanwhile, such resources are still available. Thus, unattended words would be processed in the Stroop task but not in the lexical-decision task. Lachter et al. (2004) attempted to make word-processing resources available to the irrelevant distractor words by presenting them before the target. 
However, it is possible that participants prepare for target onset by reserving word-processing resources in advance (see Lien, Ruthruff, Cornett, Goodin, \& Allen, 2008). Similarly, identification of the prime word in Lachter et al. might have been interrupted by the subsequent appearance of the target word.

In summary, the present results provide the most compelling evidence to date that some whole-word processing occurs outside the focus of spatial attention. The reported effects are small - certainly much smaller than would be expected if the words were spatially attended. However, given the theory that attention is required for word identification, it is remarkable that such effects occur at all.

\section{AUTHOR NOTE}

This research was supported by funding from NASA Grant NCC 2-1325 and from the Human Measures and Performance Project of NASA's Airspace Systems Program. We thank three dedicated reviewers, Thomas Carr, Jennifer Stolz, and James Neely, whose invaluable comments strengthened the article considerably. Correspondence concerning this article should be addressed to J. Lachter, Mail Stop 262-4, NASA Ames Research Center, Moffett Field, CA 94035 (e-mail: joel.b.lachter@nasa.gov).

\section{REFERENCES}

Besner, D., \& Stolz, J. A. (1999). What kind of attention modulates the Stroop effect? Psychonomic Bulletin \& Review, 6, 99-104.

BRown, T. L. (1996). Attentional selection and word processing in Stroop and word search tasks: The role of selection for action. American Journal of Psychology, 109, 265-286.

Brown, T. L., Gore, C. L., \& CARr, T. H. (2002). Visual attention and word recognition in Stroop color naming: Is word recognition "automatic"? Journal of Experimental Psychology: General, 131, 220-240.

Brown, T. L., Roos-Gilbert, L., \& CARr, T. H. (1995). Automaticity and word perception: Evidence from Stroop and Stroop dilution effects. Journal of Experimental Psychology: Learning, Memory, \& Cognition, 21, 1395-1411.

Cho, Y. S., Lien, M.-C., \& Proctor, R. W. (2006). Stroop dilution depends on the nature of the color carrier but not on its location. Journal of Experimental Psychology: Human Perception \& Performance, 32, 826-839.

Fera, P., Jolicceur, P., \& Besner, D. (1994). Evidence against early selection: Stimulus quality effects in previewed displays. Journal of Experimental Psychology: Human Perception \& Performance, 20, 259-275.

LAChter, J., Forster, K. I., \& RUTHRUfF, E. (2004). Forty-five years after Broadbent (1958): Still no identification without attention. Psychological Review, 111, 880-913.
Lien, M.-C., Ruthruff, E., Cornett, L., Goodin, Z., \& Allen, P. A. (2008). On the nonautomaticity of visual word processing: Electrophysiological evidence that word processing requires central attention. Journal of Experimental Psychology: Human Perception \& Performance, 34, 751-773.

MacLEOD, C. M. (1991). Half a century of research on the Stroop effect: An integrative review. Psychological Bulletin, 109, 163-203.

McCann, R. S., Folk, C. L., \& Johnston, J. C. (1992). The role of spatial attention in visual word processing. Journal of Experimental Psychology: Human Perception \& Performance, 18, 1015-1029.

NeELY, J. H., \& KaHAN, T. A. (2001). Is semantic activation automatic? A critical re-evaluation. In H. L. Roediger III, J. S. Nairne, I. Neath, \& A. M. Surprenant (Eds.), The nature of remembering: Essays in honor of Robert G. Crowder (pp. 69-93). Washington, DC: American Psychological Association.

Posner, M. I., Snyder, C. R. R., \& Davidson, B. J. (1980). Attention and the detection of signals. Journal of Experimental Psychology: General, 109, 160-174.

Stroop, J. R. (1935). Studies of interference in serial verbal reactions. Journal of Experimental Psychology, 18, 643-662.

Treisman, A. (1960). Contextual cues in selective listening. Quarterly Journal of Experimental Psychology, 12, 242-248.

YAnTis, S., \& Johnston, J. C. (1990). On the locus of visual selection: Evidence from focused attention tasks. Journal of Experimental Psychology: Human Perception \& Performance, 16, 135-149.

\section{NOTES}

1. Posner, Snyder, and Davidson (1980) found that presenting the target in a fixed location (allowing endogenous attention) was less effective at manipulating attention than was cuing the target location (exogenously). Here, we did both. It is conceivable, however, that varying the location of the target might lead to an even stronger focus of attention than that which was achieved here (see Yantis \& Johnston, 1990, p. 138, for a discussion of this issue).

2. This pilot study used stimuli identical to those of the present Experiment $2 \mathrm{~A}$, except that the exogenous and endogenous cues focusing attention on the target were removed. More specifically, the flashing, colored rectangles shown in Figure 1 were removed, and filler trials were added in which the target appeared at the top or bottom location.

3. The clearest results from Lachter et al. (2004) come from Experiment 5 , in which both exogenous and endogenous cues were used to focus attention away from the distractor word. Under these conditions, no priming effect was found $(-1 \mathrm{msec})$. Since that article was published, we have conducted two other (as yet unpublished) experiments that included the same conditions. In these experiments, nonsignificant trends of -2 and -4 msec were found. Across the three experiments, with 112 participants, there was a $-2.7-\mathrm{msec}$ effect of unattended words, with a standard error of $2.3 \mathrm{msec}$.

(Manuscript received March 23, 2006; revision accepted for publication April 4, 2008.) 